\title{
Estimation of a nonparametric regression spectrum for multivariate time series
}

\author{
Jan Beran \\ and \\ Mark A. Heiler \\ Department of Mathematics and Statistics \\ University of Konstanz
}

December 2007

\begin{abstract}
Estimation of a nonparametric regression spectrum based on the periodogram is considered. Neither trend estimation nor smoothing of the periodogram are required. Alternatively, for cases where spectral estimation of phase shifts fails and the shift does not depend on frequency, a time domain estimator of the lag-shift is defined. Asymptotic properties of the frequency and time domain estimators are derived. Simulations and a data example illustrate the methods.
\end{abstract}

Key words: Periodogram, cross spectrum, regression spectrum, phase, wavelets.

\section{Introduction}

Consider a multivariate time series $\mathbf{Y}(i)=\left(Y_{1}(i), \ldots, Y_{p}(i)\right)^{T}$ of the form

$$
\mathbf{Y}(i)=\mathbf{f}\left(t_{i}\right)+\epsilon(i)
$$

where $t_{i}=i / n(i=1, \ldots, n), \mathbf{f}(t)=\left(f_{1}(t), \ldots, f_{p}(t)\right)^{T} \in \mathbb{C}^{p}(t \in \mathbb{R})$ is a multivariate deterministic trend function and $\epsilon(i)=\left(\varepsilon_{1}(i), \ldots, \varepsilon_{p}(i)\right)^{T}$ is a zero 
mean stationary process. In this model, dependence between two components $Y_{r}$ and $Y_{s}$ can occur due to two reasons: 1. dependence between $\epsilon_{r}$ and $\epsilon_{s}$, and 2. dependence due to similarities in the underlying deterministic components $f_{r}$ and $f_{s}$. In the first case, linear dependence is characterized by crosscorrelations, the cross-spectrum, coherency and phase-shift between $\epsilon_{r}$ and $\epsilon_{s}$ (see e.g. standard books such as Priestley 1981, Brockwell and Davis 1987). For the second case, Beran and Heiler (2007) introduced a nonparametric regression cross spectrum. In the present paper, we consider estimation of the regression cross spectrum based on the periodogram, and frequency and time domain estimation of possible phase-shifts. Figure 5 shows a typical example where the nonparametric regression spectrum leads to interesting insights. The bivariate series consists of the Southern Oscillation Index (figure 5a) and recruitment of new fish in the central Pacific Ocean (figure 5b), ranging from 1950 to 1987 over a period of $n=453$ months (Shumway and Stoffer 2000). Both series have strong deterministic components that are related to each other. The analysis in section 5.2 shows that there are two levels of dependencies, namely between the long-term trends (El Niño effect) of both series, and between the deterministic seasonal components respectively.

The paper is organized as follows. Basic definitions from Beran and Heiler (2007) are summarized briefly in section 2. An estimator of the regression spectrum, its modulus and the phase spectrum, based on the periodogram, is discussed in section 3 , together with asymptotic properties. The asymptotic results imply in particular that phase estimates can be highly unreliable for frequencies with low amplitude spectrum. In fact, examples in section 5 illustrate that estimation of time-delays from the raw plot of the (estimated) regression phase spectrum is virtually impossible. The problem is resolved by applying an algorithm that downweighs or eliminates unreliable frequencies. For cases where the number of relevant frequencies is too small, an alternative procedure for estimating time shifts between trend functions is presented in section 4. Simulations and a data example in section 5 illustrate the methods. Proofs are given in the appendix. 


\section{Definition of the regression cross covari- ance and spectrum}

Under suitable regularity assumptions on f, Beran and Heiler (2007) define the regression (cross-)covariance function $\boldsymbol{\Gamma}(u)=\left[\gamma_{r s}(u)\right]_{r, s=1, \ldots, p}$ and the regression (cross-) correlation function $\mathbf{R}(u)=\left[\rho_{r s}(u)\right]_{r, s=1, \ldots, p}$ of $\mathbf{f}(t)$ by

$$
\gamma_{r s}(u)=<f_{r}(\cdot+u), f_{s}>=\int_{0}^{1} f_{r}(t+u) \overline{f_{s}(t)} d t
$$

and

$$
\rho_{r s}(u)=\frac{\gamma_{r s}(u)}{\sqrt{\gamma_{r}(0) \gamma_{s}(0)}}
$$

where $\int_{o}^{1} f(x) d x$ is assumed to be zero and $\mathbf{f}(1+u)=\mathbf{f}(u)(0<u \leq 1)$. Note that here, trend components that cannot be extended periodically beyond $t=1$ are assumed to have been removed, or to be negligible. For $t \in[0,1]$ and $f_{r} \in L^{2}[0,1]$, we have

$$
\mathbf{f}(t)=\sum_{j=-\infty}^{\infty} \mathbf{a}(j) e^{i 2 \pi j t}
$$

where $\mathbf{a}(j)=\left(a_{1}(j), a_{2}(j), \ldots, a_{p}(j)\right)^{T} \in \mathbb{C}^{p}$ are given by

$$
a_{r}(j)=<f_{r}, e^{i 2 \pi j \cdot}>=\int_{0}^{1} f_{r}(t) e^{-i 2 \pi j t} d t .
$$

Hence, the regression spectrum at frequency $j$ is defined as the sequence of $p \times p$ matrices $\mathbf{H}(j)=\left[h_{r s}(j)\right]_{r, s=1, \ldots, p}(j \in \mathbb{Z})$ with

$$
\mathbf{H}(j)=\mathbf{a}(j) \overline{\mathbf{a}^{T}(j)} .
$$

The regression spectrum and covariance function are closely linked by

$$
\boldsymbol{\Gamma}(u)=\sum_{j=-\infty}^{\infty} \mathbf{H}(j) e^{i 2 \pi j u}
$$

and

$$
\mathbf{H}(j)=\int_{-\frac{1}{2}}^{\frac{1}{2}} e^{-i 2 \pi j u} \boldsymbol{\Gamma}(u) d u .
$$


Using polar representation of $\mathbf{H}(j)$,

$$
\tilde{h}_{r s}(j)=\frac{h_{r s}(j)}{\sqrt{\gamma_{r r}(0) \cdot \gamma_{s s}(0)}}=\frac{\left|a_{r}(j) \overline{a_{s}(j)}\right|}{\sqrt{\gamma_{r r}(0) \cdot \gamma_{s s}(0)}} \exp \left(i \phi_{r s}(j)\right)
$$

is called the standardized regression spectrum of $\mathbf{f}$,

$$
\begin{aligned}
\kappa_{r s}(j) & =\frac{\left|h_{r s}(j)\right|}{\sqrt{\gamma_{r r}(0) \cdot \gamma_{s s}(0)}}=\frac{\left|h_{r s}(j)\right|}{|| f_{r}|| \cdot|| f_{s} \|} \\
& =\frac{\left|a_{r}(j) \overline{a_{s}(j)}\right|}{\sqrt{\sum_{l}\left|a_{r}(l)\right|^{2} \sum_{m}\left|a_{s}(m)\right|^{2}}}
\end{aligned}
$$

is the relative spectral modulus and $\phi_{r s}(j)$ the phase shift at frequency $j$. The relative spectral modulus (or coherence) $\kappa_{r s}(j)$ defined above can assume any number between 0 and 1 , thus giving a relative measure of the contribution of frequency $j$ to the cross-covariance.

Remark 1 If two components $f_{r}$ and $f_{s}$ are shifted versions of each other, i.e.

$$
f_{s}(t)=c \cdot f_{r}(t+\Delta)
$$

for some $\Delta, c \in \mathbb{R}$, then

$$
\kappa_{r s}(j)=\frac{\left|a_{r}(j)\right|^{2}}{\sum_{l}\left|a_{r}(l)\right|^{2}}
$$

and the phase-shift

$$
\phi_{r s}(j)=-2 \pi \Delta j
$$

is a linear function of the shift parameter $\Delta$.

\section{Estimation in the spectral domain}

\subsection{The periodogram}

In practice, the trend component $\mathbf{f}$ is usually unknown. Beran and Heiler (2007) propose an estimator of the cross-spectrum based on a trend estimator $\hat{\mathbf{f}}$ obtained by wavelet thresholding. In this section, we consider a direct estimator of the regression spectrum based on he periodogram. This has two

main advantages. First of all, the estimator is simple and does not require 
nonparametric estimation of the trend function $\mathbf{f}$. The second advantage is that estimated values at different frequencies are asymptotically independent.

Given $n$ observations of a multivariate vector $\mathbf{Y}(i)(i=1, \ldots, n)$, the periodogram of $\mathbf{Y}(i)$ at frequency $\omega_{j}=2 \pi j / n, \omega_{j} \in[-\pi, \pi]$, is defined by

$$
\mathbf{I}\left(\omega_{j}\right)=\frac{1}{n}\left(\sum_{s=1}^{n} \mathbf{Y}(s) \exp \left(-i \omega_{j} s\right)\right)\left(\sum_{t=1}^{n} \mathbf{Y}(t) \exp \left(i \omega_{j} t\right)\right)^{T} .
$$

Moreover, let

$$
\mathbf{A}\left(\omega_{j}\right)=\sum \mathbf{f}\left(t_{k}\right) \exp \left(-i \omega_{j} k\right)
$$

and

$$
\mathbf{B}\left(\omega_{j}\right)=\sum \epsilon(k) \exp \left(-i \omega_{j} k\right)
$$

respectively. Then

$$
\mathbf{I}_{\epsilon}\left(\omega_{j}\right)=\left[I_{\epsilon ; r s}\left(\omega_{j}\right)\right]_{1 \leq r, s \leq p}=\frac{1}{n} \mathbf{B}\left(\omega_{j}\right){\overline{\mathbf{B}\left(\omega_{j}\right)}}^{T},
$$

is the periodogram of the multivariate stationary series $\epsilon(i)=\left(\epsilon_{1}(i), \ldots, \epsilon_{p}(i)\right)^{T}$. The deterministic counterpart,

$$
\mathbf{I}_{\mathbf{f}}\left(\omega_{j}\right)=\left[I_{f ; r s}\left(\omega_{j}\right)\right]_{1 \leq r, s \leq p}=\frac{1}{n} \mathbf{A}\left(\omega_{j}\right){\overline{\mathbf{A}\left(\omega_{j}\right)}}^{T}
$$

will be called regression periodogram of $\mathbf{f}$. It can be seen by straightforward calculations that under model (1) with non-constant $\mathbf{f}$ the diagonal elements of the periodogram $\mathbf{I}\left(\omega_{j}\right)$ are of the order $O(n)$ and are dominated by $\mathbf{I}_{\mathbf{f}}\left(\omega_{j}\right)$. This is the essential reason why the regression spectrum can be estimated directly from the periodogram. Specific results on the asymptotic distribution are given in the following two theorems.

Theorem 1 Denote by $\mathbf{H}=\left[h_{r s}\right]_{r, s=1, \ldots, p}$ the regression spectrum of $\mathbf{f}$, and suppose that $\epsilon(i)$ are independent, identically zero mean random vectors with non-singular covariance matrix $\boldsymbol{\Sigma}=\left(\Sigma_{r s}\right)_{1 \leq r, s \leq p}$, and existing fourth moments. Then, for each pair $(r, s)$, and Fourier frequencies $0 \leq \omega_{j_{1}}<\cdots<$ $\omega_{j_{k}} \leq \pi,(k \in \mathbb{N})$, the following holds.

(i) $n^{-1} I_{r s}\left(\omega_{j}\right)-h_{r s}(j)=O_{p}\left(n^{-1 / 2}\right)$,

(ii) $E\left[n^{-1} I_{r s}\left(\omega_{j}\right)\right]-h_{r s}(j)=O\left(n^{-1}\right)$, 
(iii)

$$
\sqrt{n}\left[n^{-1} I_{r s}\left(\omega_{j_{1}}\right)-h_{r s}\left(j_{1}\right), \ldots, n^{-1} I_{r s}\left(\omega_{j_{k}}\right)-h_{r s}\left(j_{k}\right)\right]^{T}
$$

converges in distribution to a $k$-dimesional normal random vector with mean $\mathbf{0}$ and covariances

$$
\lim _{n \rightarrow \infty} n \operatorname{cov}\left(n^{-1} I_{r s}\left(\omega_{j}\right), n^{-1} I_{r s}\left(\omega_{j^{\prime}}\right)\right)=0,
$$

for $\omega_{j} \neq \omega_{j^{\prime}}$, and

$$
\lim _{n \rightarrow \infty} n \operatorname{var}\left(n^{-1} I_{r s}\left(\omega_{j}\right)\right)=\Sigma_{r r}\left|a_{s}(j)\right|^{2}+\Sigma_{s s}\left|a_{r}(j)\right|^{2} .
$$

Remark 2 More specifically, the proof of the theorem implies

$$
n \operatorname{cov}\left(n^{-1} I_{r s}\left(\omega_{j}\right), n^{-1} I_{r s}\left(\omega_{j^{\prime}}\right)\right)=O\left(n^{-2}\right),
$$

for $\omega_{j} \neq \omega_{j^{\prime}}$, and

$$
n \operatorname{var}\left(n^{-1} I_{r s}\left(\omega_{j}\right)\right)=\Sigma_{r r}\left|a_{s}(j)\right|^{2}+\Sigma_{s s}\left|a_{r}(j)\right|^{2}+R_{n}
$$

with $\Sigma_{r s}$ denoting the $(r, s)$ th entry of the matrix $\Sigma$ and

$$
R_{n}=n^{-1} \Sigma_{r r} \Sigma_{s s}+O\left(n^{-2}\right)
$$

for $0<\omega_{j}<\pi$, and

$$
R_{n}=n^{-1}\left(\Sigma_{r r} \Sigma_{s s}+\Sigma_{r s} \Sigma_{s r}\right)+O\left(n^{-2}\right)
$$

for $\omega_{j} \in\{0, \pi\}$.

Remark 3 The periodogram $\mathbf{I}(\omega)$ contains a stochastic and a deterministic part. This carries over to the asymptotic variance at frequency $j$. The main component is given by a product of the variance of the stationary part and the regression spectrum. The variance in theorem 1 is of order $O\left(n^{-1}\right)$ so that $n^{-1} I_{r s}\left(\omega_{j}\right)$ is an asymptotically consistent estimator of $h_{r s}(j)$. This is in contrast to spectral estimation for stationary processes where the periodogram needs to be smoothed. Note also that, in contrast to the wavelet estimator in Beran and Heiler (2007), the estimators $\hat{\mathbf{f}}(\omega)=n^{-1} \mathbf{I}(\omega)$ at different frequencies are asymptotically independent. This facilitates estimation of the coherence and phase spectrum (see results below). 
Theorem 1 can now be extended to linear error processes. Thus, assume

$$
\epsilon(i)=\sum_{j=-\infty}^{\infty} \mathbf{A}(j) \mathbf{Z}(i-j),
$$

where $\mathbf{A}(j)=\left(A_{l k}(j)\right)_{1 \leq l, k \leq p}$ are $p \times p$-matrices such that for all pairs $(l, k)$,

$$
\sum_{j \in \mathbb{Z}}\left|A_{l k}(j)\right||j|^{\frac{1}{2}}<\infty
$$

and $\mathbf{Z}(i)$ are independent identically distributed with zero mean and nonsingular covariance matrix $\boldsymbol{\Sigma}$. Also, denote by

$$
\mathbf{h}_{\epsilon}=\left[h_{\epsilon ; r, s}\right]_{r, s=1,2, \ldots, p}
$$

the cross-spectral density of $\epsilon(i)$. Theorem 1 can be generalized to

Theorem 2 Let $\epsilon(i)$ be a linear process as defined above. Then, with the same notation as in theorem 1,

(i) $n^{-1} I_{r s}\left(\omega_{j}\right)-h_{r s}(j)=O_{p}\left(n^{-1 / 2}\right)$;

(ii) $E\left[n^{-1} I_{r s}\left(\omega_{j}\right)\right]-h_{r s}(j)=O\left(n^{-1}\right)$;

(iii)

$$
\sqrt{n}\left[n^{-1} I_{r s}\left(\omega_{j_{1}}\right)-h_{r s}\left(j_{1}\right), \ldots, n^{-1} I_{r s}\left(\omega_{j_{k}}\right)-h_{r s}\left(j_{k}\right)\right]^{T}
$$

converges in distribution to a $k$-dimensional normal random variable with mean zero,

$$
\begin{gathered}
\lim _{n \rightarrow \infty} n \operatorname{cov}\left(n^{-1} I_{r s}\left(\omega_{j}\right), n^{-1} I_{r s}\left(\omega_{j^{\prime}}\right)\right)=0 \\
\text { for } \omega_{j} \neq \omega_{j^{\prime}} \text {, and } \\
\lim _{n \rightarrow \infty} n \operatorname{var}\left(n^{-1} I_{r s}\left(\omega_{j}\right)\right)=2 \pi h_{\epsilon ; r r}\left(\omega_{j}\right)\left|a_{s}(j)\right|^{2}+2 \pi h_{\epsilon ; s s}\left(\omega_{j}\right)\left|a_{r}(j)\right|^{2} .
\end{gathered}
$$

Remark 4 More specifically, the proof of the theorem implies

$$
n \operatorname{cov}\left(n^{-1} I_{r s}\left(\omega_{j}\right), n^{-1} I_{r s}\left(\omega_{j^{\prime}}\right)\right)=O\left(n^{-2}\right)
$$




$$
\begin{aligned}
& \text { for } \omega_{j} \neq \omega_{j^{\prime}} \text {, and } \\
& n \operatorname{var}\left(n^{-1} I_{r s}\left(\omega_{j}\right)\right)=2 \pi h_{\epsilon ; r r}\left(\omega_{j}\right)\left|a_{s}(j)\right|^{2}+2 \pi h_{\epsilon ; s s}\left(\omega_{j}\right)\left|a_{r}(j)\right|^{2}+R_{n}
\end{aligned}
$$

with

$$
R_{n}=n^{-1}(2 \pi)^{2} h_{\epsilon ; r r}\left(\omega_{j}\right) h_{\epsilon ; s s}\left(\omega_{j}\right)+O\left(n^{-2}\right)
$$

for $0<\omega_{j}<\pi$, and

$$
R_{n}=n^{-1}(2 \pi)^{2}\left[h_{\epsilon ; r r}\left(\omega_{j}\right) h_{\epsilon ; s s}\left(\omega_{j}\right)+h_{\epsilon ; r s}\left(\omega_{j}\right) h_{\epsilon ; s r}\left(\omega_{j}\right)\right]
$$

for $\omega_{j} \in\{0, \pi\}$.

Remark 5 Recalling that $h_{r s}(j)=a_{r}(j) \overline{a_{s}(j)}$, we see that the asymptotic variance of $I_{r s}\left(\omega_{j}\right)$ is equal to $2 \pi$ times the product of the regression and the stationary spectrum.

Remark 6 The result in theorem 2 can be generalized to pairs $(r, s)$ and $\left(r^{\prime}, s^{\prime}\right), 1 \leq r, s, r^{\prime}, s^{\prime} \leq p$. For Fourier frequencies $\omega_{j}, \omega_{j^{\prime}}$ we have

$$
\lim _{n \rightarrow \infty} n \operatorname{cov}\left(n^{-1} I_{r s}\left(\omega_{j}\right), n^{-1} I_{r^{\prime} s^{\prime}}\left(\omega_{j^{\prime}}\right)\right)=O\left(n^{-2}\right)
$$

for $j \neq j^{\prime}$ and

$\lim _{n \rightarrow \infty} n \operatorname{cov}\left(n^{-1} I_{r s}\left(\omega_{j}\right), n^{-1} I_{r^{\prime} s^{\prime}}\left(\omega_{j^{\prime}}\right)\right)=2 \pi \overline{h_{s s^{\prime}}(j)} h_{\epsilon ; r r^{\prime}}\left(\omega_{j}\right)+2 \pi \overline{h_{r r^{\prime}}(j)} h_{\epsilon ; s s^{\prime}}\left(\omega_{j}\right)+O\left(n^{-1}\right)$

for $j=j^{\prime}$.

\subsection{Estimation of modulus, phase spectrum and phase shift}

We now consider estimation of the modulus and the phase spectrum. As a special case, the asymptotic results will be applied to the estimation of a constant phase shift. Denote by

$$
c_{r s}(j)=\mathfrak{R e}\left\{h_{r s}(j)\right\}
$$

and

$$
q_{r s}(j)=-\mathfrak{I m}\left\{h_{r s}(j)\right\}
$$


the real part and the imaginary part with reversed sign of $h_{r s}(j)=a_{r}(j) \overline{a_{s}(j)}$ respectively. Thus,

$$
h_{r s}(j)=c_{r s}(j)-i q_{r s}(j) .
$$

Similarily, we define for the cross spectral density of $\varepsilon(i)$ the quantities

$$
c_{\varepsilon ; r s}(j)=\mathfrak{R e}\left\{h_{\varepsilon ; r s}(j)\right\}
$$

and

$$
q_{\varepsilon ; r s}(j)=-\mathfrak{I m}\left\{h_{\varepsilon ; r s}(j)\right\} .
$$

The joint asymptotic distribution of $\hat{c}_{r s}(j), \hat{q}_{r s}(j)$ follows directly from theorem 1 and remark 6 .

Lemma 1 Define the estimators

$$
\hat{c}_{r s}(j)=\frac{1}{2 n}\left[I_{r s}\left(\omega_{j}\right)+\overline{I_{r s}\left(\omega_{j}\right)}\right]
$$

and

$$
\hat{q}_{r s}(j)=-\frac{1}{2 n i}\left[I_{r s}\left(\omega_{j}\right)-\overline{I_{r s}\left(\omega_{j}\right)}\right]
$$

Then,

$$
\zeta_{n}=\sqrt{n}\left[\hat{c}_{r s}(j)-c_{r s}(j), \hat{q}_{r s}(j)-q_{r s}(j)\right]^{T}
$$

converges in distribution to a bivariate normal zero mean random variable with asymptotic covariance matrix $M(j)=\left[M_{i j}(j)\right]_{i, j=1,2}$ given by

$$
\begin{aligned}
M_{11}(j) & =\lim _{n \rightarrow \infty} n \operatorname{var}\left(\hat{c}_{r s}(j)\right) \\
& =\pi\left[h_{s s}(j) h_{\epsilon ; r r}\left(\omega_{j}\right)+h_{r r}(j) h_{\epsilon ; s s}\left(\omega_{j}\right)\right]+2 \pi\left[c_{r s}(j) c_{\epsilon ; r s}\left(\omega_{j}\right)-q_{r s}(j) q_{\epsilon ; r s}\left(\omega_{j}\right)\right], \\
M_{22}(j) & =\lim _{n \rightarrow \infty} n \operatorname{var}\left(\hat{q}_{r s}(j)\right) \\
& =\pi\left[h_{s s}(j) h_{\epsilon ; r r}\left(\omega_{j}\right)+h_{r r}(j) h_{\epsilon ; s s}\left(\omega_{j}\right)\right]-2 \pi\left[c_{r s}(j) c_{\epsilon ; r s}\left(\omega_{j}\right)-q_{r s}(j) q_{\epsilon ; r s}\left(\omega_{j}\right)\right],
\end{aligned}
$$

and

$$
\begin{aligned}
M_{12}(j) & =\lim _{n \rightarrow \infty} n \operatorname{cov}\left(\hat{c}_{r s}(j), \hat{q}_{r s}(j)\right) \\
& =2 \pi\left[q_{r s}(j) c_{\epsilon ; r s}\left(\omega_{j}\right)+c_{r s}(j) q_{\epsilon ; r s}\left(\omega_{j}\right)\right] .
\end{aligned}
$$


Based on lemma 1 we may now construct consistent estimators of the spectral modulus and the phase shift. Using the notation

$$
h_{r s}(j)=\kappa_{r s}^{*}(j) \exp \left(i \phi_{r s}(j)\right)
$$

estimators of the spectral modulus and phase shift respectively are defined by

$$
\hat{\kappa}_{r s}^{*}(j)=\sqrt{\hat{c}_{r s}^{2}(j)+\hat{q}_{r s}^{2}(j)}
$$

and by

$$
\hat{\phi}_{r s}(j)=\arg I_{r s}\left(\omega_{j}\right)=\arctan \left(-\frac{\hat{q}_{r s}(j)}{\hat{c}_{r s}(j)}\right) .
$$

The asymptotic distribution of $\hat{\kappa}_{r s}^{*}(j)$ and $\hat{\phi}_{r s}(j)$ is given in the following corollaries.

Corollary 1 Let $\kappa_{r s}^{*}(j)>0$ and let the assumptions of theorem 2 hold, then

$$
\hat{\kappa}_{r s}^{*}(j)-\kappa_{r s}^{*}(j)=O_{p}\left(n^{-1 / 2}\right)
$$

uniformly for all $j \in \mathbb{Z}$. Furthermore,

$$
\sqrt{n}\left[\hat{\kappa}_{r s}^{*}(j)-\kappa_{r s}^{*}(j)\right] \stackrel{d}{\rightarrow} \mathcal{N}\left(0, \sigma_{\kappa ; r s}^{2}(j)\right),
$$

where

$$
\sigma_{\kappa ; r s}^{2}(j)=\frac{c_{r s}^{2}(j) M_{11}(j)+q_{r s}^{2}(j) M_{22}(j)+2 c_{r s}(j) q_{r s}(j) M_{12}(j)}{\left[\kappa_{r s}^{*}(j)\right]^{2}} .
$$

Moreover,

$$
\lim _{n \rightarrow \infty} n \operatorname{cov}\left(\hat{\kappa}_{r s}^{*}(j), \hat{\kappa}_{r s}^{*}\left(j^{\prime}\right)\right)=O\left(n^{-2}\right)\left(j \neq j^{\prime}\right)
$$

Corollary 2 Let $\kappa_{r s}^{*}(j)>0$ with $\phi_{r s}(j)=\arg h_{r s}(j)$ and $\hat{\phi}_{r s}(j)$ as above. Then,

$$
\hat{\phi}_{r s}(j)-\phi_{r s}(j)=O_{p}\left(n^{-1 / 2}\right)
$$

uniformly for all $j \in \mathbb{Z}$. Furthermore,

$$
\sqrt{n}\left[\hat{\phi}_{r s}(j)-\phi_{r s}(j)\right] \stackrel{d}{\rightarrow} \mathcal{N}\left(0, \sigma_{\phi ; r s}^{2}(j)\right),
$$


where

$$
\sigma_{\phi ; r s}^{2}(j)=\frac{q_{r s}^{2}(j) M_{11}(j)+c_{r s}^{2}(j) M_{22}(j)-2 c_{r s}(j) q_{r s}(j) M_{12}(j)}{\left[\kappa_{r s}^{*}(j)\right]^{4}} .
$$

Moreover,

$$
\lim _{n \rightarrow \infty} n \operatorname{cov}\left(\hat{\phi}_{r s}(j), \hat{\phi}_{r s}\left(j^{\prime}\right)\right)=O\left(n^{-2}\right)\left(j \neq j^{\prime}\right)
$$

Note that the variance of the phase spectrum is small whenever the modulus $\kappa_{r s}^{*}(j)$ is large and vice versa. Accurate estimation of the phase spectrum may therefore only be expected for frequencies $j$ where the amplitude spectrum is large. Examples in section 5 illustrate that often most frequencies have to be omitted in the estimation of phase shifts. For instance, in the case of a simple shift between $f_{r}$ and $f_{s}$, i.e.

$$
f_{r}(t)=c f_{s}(t+\Delta)
$$

and hence

$$
\phi_{r s}(j)=-2 \pi j \Delta,
$$

the following algorithm can be applied:

1. Calculate $\hat{\mathbf{f}}\left(\omega_{j}\right)=n^{-1} \mathbf{I}\left(\omega_{j}\right)$;

2. Define

$$
J^{*}=\left\{j: \hat{\kappa}_{r s}^{*}(j)>c \cdot \sqrt{\sigma_{\kappa ; r s}^{2}(j)}\right\}
$$

for a suitably chosen $c \in \mathbb{R}$;

3. Estimate the phase shift by applying a local robust regression to the points $\left\{\left(j, \phi_{r s}(j)\right): j \in J^{*}\right\}$, taking into account possible jumps modulo $2 \pi$.

\section{Lag Estimation in the time domain}

In the previous sections we considered estimation of the regression cross spectrum based on the periodogram and derived a method for estimating lead-lag effects in the trend components. The proposed algorithm is based on a set of significant common frequencies that can be used for estimating the slope of the phase line. Problems with this algorithm are expected if 
the set of common frequencies is too small to identify a slope in the phase plot. This is the case, for instance, if the deterministic components have a Fourier series representation with a small number of harmonic components. Phase-shifts may then have to be identified by examining regression cross correlations instead of the phase spectrum. In the case of a simple shift that does not depend on frequency, the time delay between two trend components can be estimated by identifying the maximum of the cross correlation.

Thus, for each pair $(r, s), 1 \leq r, s \leq p$, denote the set of local maxima by

$$
\mathcal{M}=\left\{u \in[-1,1]: \gamma_{r s}^{\prime}(u)=0, \gamma_{r s}^{\prime \prime}(u)<0\right\}
$$

and

$$
u_{r s}^{\max }=\operatorname{argmax}\left\{\gamma_{r s}(u): u \in \mathcal{M}\right\}
$$

where $\gamma_{r s}$ is the cross autocorrelation defined in section 1. An estimator of $u_{r s}^{\max }$ is then defined by

$$
\hat{u}_{r s}^{\max }=\operatorname{argmax}\left\{\hat{\gamma}_{r s}(u): u \in \hat{\mathcal{M}}\right\}
$$

where

$$
\hat{\mathcal{M}}=\left\{u \in[-1,1]: \hat{\gamma}_{r s}^{\prime}(u)=0, \hat{\gamma}_{r s}^{\prime \prime}(u)<0\right\}
$$

and $\hat{\gamma}_{r s}$ is a suitable consistent estimator of $\gamma_{r s}(u)$.

More specifically, here $\hat{\gamma}_{r s}$ will be defined using a wavelet estimator of the trend function $\mathbf{f} \in \mathbb{R}^{p}$ in the definition of $\gamma_{r s}$. Thus, given a father and mother wavelet $\phi(\cdot), \psi(\cdot) \in L^{2}(\mathbb{R})$ and the corresponding wavelet basis

$$
\phi_{l, k}(x)=2^{\frac{l}{2}} \phi\left(2^{l} x-k\right)
$$

and

$$
\psi_{j, k}(x)=2^{\frac{j}{2}} \psi\left(2^{j} x-k\right), \quad k, j \in \mathbb{Z},
$$

we define

$$
\hat{\gamma}_{r s}(u)=\int_{0}^{1} \hat{f}_{r}(t+u) \hat{f}_{s}(t) d t
$$

with

$$
\hat{f}_{r}(t):=\sum_{k} \hat{\alpha}_{l, k}^{(r)} \phi_{l, k}(t)+\sum_{j \geq l}^{J_{n}} \sum_{k} \hat{w}_{j, k}^{(r)} \hat{\beta}_{j, k}^{(r)} \psi_{j, k}(t),
$$

where

$$
\hat{\alpha}_{l, k}^{(r)}=\frac{1}{n} \sum_{u=1}^{n} \phi_{l, k}\left(t_{u}\right) Y_{r}(u)
$$


and

$$
\hat{\beta}_{j, k}^{(r)}=\frac{1}{n} \sum_{u=1}^{n} \psi_{j, k}\left(t_{u}\right) Y(u),
$$

for some $J_{n} \rightarrow \infty$, and $\hat{w}_{j, k}^{(r)}:=\mathbf{1}\left\{\left|\hat{\beta}_{j, k}^{(r)}\right| \geq \sqrt{\operatorname{var}\left(\hat{\beta}_{j, k}^{(r)}\right)} \lambda_{j}\right\}$. For the choice of the threshold $\lambda_{j}$ see e.g. Brillinger $(1994,1996)$ and Donoho and Johnston (1995), among others. Denote by $\boldsymbol{\alpha}^{(r)}=\left\{\alpha_{l, k}^{(r)}: l, k \in \mathbb{Z}, \alpha_{l, k}^{(r)} \neq 0\right\}$ and $\boldsymbol{\beta}^{(r)}=\left\{\beta_{j, k}^{(r)}: j \geq l, k \in \mathbb{Z}, \beta_{j, k}^{(r)} \neq 0\right\}(r=1, \ldots, p)$ the coefficients in the wavelet representation of the components of $\mathbf{f}$, i.e.

$$
f_{r}(t):=\sum_{k} \alpha_{l, k}^{(r)} \phi_{l, k}(t)+\sum_{j \geq l}^{J_{n}} \sum_{k} \beta_{j, k}^{(r)} \psi_{j, k}(t) .
$$

As in Brillinger (1995), we assume that for each $r$, the number of non-zero coefficients $\alpha_{l, k}^{(r)}$ and $\beta_{j, k}^{(r)}$ is finite. Let $r, s \in\{1,2, \ldots, p\}$ be fixed. Defining

$$
\theta_{0}=\left(\boldsymbol{\alpha}^{(r)}, \boldsymbol{\beta}^{(r)}, \boldsymbol{\alpha}^{(s)}, \boldsymbol{\beta}^{(s)}\right)
$$

we may then write

$$
\gamma_{r s}(u)=\gamma_{r s}\left(u, \theta_{0}\right)
$$

where $\gamma_{r s}$ depends continuously on $\theta_{o}$,

$$
\mathcal{M}=\mathcal{M}\left(\theta_{0}\right)=\left\{u \in[-1,1]: \gamma_{r s}^{\prime}\left(u, \theta_{0}\right)=0, \gamma_{r s}^{\prime \prime}\left(u, \theta_{0}\right)<0\right\}
$$

and

$$
u_{r s}^{\max }=\operatorname{argmax}\left\{\left|\gamma_{r s}\left(u, \theta_{0}\right)\right|: u \in \mathcal{M}\left(\theta_{0}\right)\right\} .
$$

The estimator of $u_{r s}^{\max }$ is then defined by

$$
\hat{u}_{r s}^{\max }=\operatorname{argmax}\left\{\left|\gamma_{r s}(u, \hat{\theta})\right|: u \in \mathcal{M}(\hat{\theta})\right\} .
$$

To ensure existence, uniqueness and consistency of estimator the following assumptions will be used.

(A1) $\phi, \psi$ have compact support and are of finite variation;

(A2) $f_{r}(r=1,2, \ldots, p)$ are as defined in section 1 and of bounded variation; 
(A3) The cumulants

$$
c_{m ; r}\left(u_{1}, \ldots, u_{m-1}\right)=\operatorname{cum}\left\{\varepsilon_{r}\left(i+u_{1}\right), \ldots, \varepsilon_{r}\left(i+u_{m-1}\right), \varepsilon_{r}(i)\right\}
$$

of $\varepsilon_{r}(i)$ exist, are absolutely summable, i.e.

$$
C_{m ; r}=\sum_{u_{1}, \ldots, u_{m-1}}\left|c_{m ; r}\left(u_{1}, \ldots, u_{m-1}\right)\right|<\infty .
$$

Moreover, $\varepsilon_{r}$ has covariances $\gamma_{\varepsilon_{r}}(k)$ such that

$$
\sum_{k=-\infty}^{\infty}\left|k \gamma_{\varepsilon_{r}}(k)\right|<\infty
$$

(A4) $\operatorname{dim}\left(\theta_{0}\right)<\infty$

(A5) For $z$ in a small neighborhood of 0 ,

$$
\sum_{m} C_{m} z^{m}<\infty
$$

(A6) As $n \rightarrow \infty$, we have $J_{n} \rightarrow \infty, n 2^{-J_{n} / 2} \rightarrow \infty, 2^{j / 2} \lambda_{j}=o\left(n^{1 / 2}\right)(j=$ $\left.l, l+1, \ldots, J_{n}\right)$ and

$$
\sum_{j>l}^{J_{n}} 2^{j / 2} \exp \left(-2 \lambda_{j}^{2} /(1+\eta)\right)=o(1)
$$

for some $\eta>0$;

(A7) $\gamma_{r s}(u, \theta)$ is twice continously differentiable with respect to $u$ and $\theta$;

(A8) $\left|\gamma_{r s}\left(u, \theta_{0}\right)\right|$ has a unique maximum at $u_{r s}^{\max }$.

Asymptotic properties of $\hat{u}_{r s}^{\max }$ are given in the following theorem.

Theorem 3 Under assumptions (A1)-(A8) we have, for $1 \leq r, s \leq p$,

$$
\hat{u}_{r s}^{\max }-u_{r s}^{\max }=O_{p}\left(n^{-1 / 2}\right)
$$

and

$$
\sqrt{n}\left(\hat{u}_{r s}^{\max }-u_{r s}^{\max }\right) \stackrel{d}{\longrightarrow} \mathcal{N}\left(0, \tau_{u, r s}\right),
$$

with

$$
\tau_{u, r s}\left(\theta_{0}\right)=\frac{1}{\left(\gamma_{r s}^{\prime \prime}\left(u_{r s}^{\max }, \theta_{0}\right)\right)^{2}}\left(\frac{\partial}{\partial \theta} \gamma_{r s}^{\prime}\left(u_{r s}^{\max }, \theta_{0}\right)\right)^{T} \operatorname{var}(\hat{\theta})\left(\frac{\partial}{\partial \theta} \gamma_{r s}^{\prime}\left(u_{r s}^{\max }, \theta_{0}\right)\right)
$$




\section{Examples}

\subsection{Simulations}

Consider model (1) with $f_{1}(x)$ a piecewise constant function as displayed in figure 1c, $f_{2}(x)=f_{1}(x+\Delta)$ with $\Delta=.0625, \epsilon_{1}(i), \epsilon_{2}(i)$ independent and identically distributed $N\left(0, \sigma_{\epsilon}^{2}\right), \sigma_{\epsilon}^{2}=9$ and $\operatorname{corr}\left(\epsilon_{1}(i), \epsilon_{2}(j)\right)=0$. A simulated sample path of $\mathbf{Y}(i)=\left(Y_{1}, Y_{2}\right)^{T}(i=1,2, \ldots, 2048)$ with $Y_{j}(i)=$ $f_{j}\left(t_{i}\right)+\epsilon_{j}(i)(j=1,2)$ is displayed in figures $1 \mathrm{a}$ and $\mathrm{b}$. The regression amplitude and phase spectrum for these trend components are shown in figure 1e and f. Estimates of the regression amplitude and phase spectrum obtained from $n^{-1} \mathbf{I}$ are shown in figures $1 \mathrm{~g}$ and $\mathrm{h}$ respectively. Figures $1 \mathrm{~g}, \mathrm{~h}$ illustrate that the common frequencies can be identified quite accurately in the amplitude spectrum, whereas the phase spectrum is heavily disturbed by the random noise components $\epsilon_{1}, \epsilon_{2}$. This is expected in view of corollary 1 and 2. It is therefore essential to use important common frequencies only, when estimating the regression phase spectrum.

Figure 2a through d display results of a small simulation study where the simulated and true variance of the amplitude spectrum according to (9) are compared for different sample sizes. At each frequency $N=200$ simulations are carried out and the amplitude spectrum was estimated. The empirical standard deviations multiplied by $\sqrt{n}$ are plotted in figures $2 \mathrm{a}$ through d (black line) together with their asymptotic counterparts (red line). Convergence to the asymptotic standard deviation is apparently faster for frequencies where the amplitude spectrum is large. These are exactly that are used for estimating time shifts.

\begin{tabular}{l|c|c|c|c}
\hline \hline $\mathrm{n}$ & 512 & 1024 & 2048 & 4096 \\
\hline true value & 0.0625 & 0.0625 & 0.0625 & 0.0625 \\
median & 0.06012 & 0.06073 & 0.06322 & 0.06300 \\
mean & 0.05856 & 0.05838 & 0.06306 & 0.06262 \\
std.dev. & 0.03816 & 0.02275 & 0.00918 & 0.005528 \\
\hline
\end{tabular}

Table 1: Summary statistics of lag estimates. For each sample size, 200 simulations were carried out.

Figure 3a shows the amplitude spectrum and the phase estimate for one simulated series. Frequencies where the estimated amplitude spectrum is 
above four times its standard deviation are highlighted by black squares in figures $3 \mathrm{a}$ and $\mathrm{b}$. The resulting estimated phase line in figure $3 \mathrm{~b}$ is obtained by linear regression using these points only, taking into account jumps modulo $2 \pi$. The red lines indicate $99 \%$ confidence intervals for the regression slope. The regression line in figure $3 \mathrm{~b}$, with slope around 0.39 , is obviously very similar to the true phase spectrum (figure 1f) with slope 0.30. For a more systematic illustration of finite sample properties of $\hat{\Delta}$, a small simulation study was carried out, with sample sizes $n=512,1024,2048$ and $n=4096$. In each case we ran 200 simulations. Boxplots of $\hat{\Delta}$ (figure 4 ) based on 200 simulations (for each $n$ ) illustrate that estimation of $\Delta$ is very difficult for $n=512$. The accuracy of $\hat{\Delta}$ improves fast, however, with increasing sample size. A detailed summary of this simulation study is given in table 1.

Finally, we examine in how far confidence intervals for $\Delta$, based on weighted linear regression of $(j, \hat{\phi})$ (with weights and residual variances obtained from corollary 2) have the desired coverage probability. For each simulated series, the six frequencies with largest cross-spectral modulus were used in the regression, and 95\%-confidence intervals were calculated using estimated variances of $\hat{\phi}$ at these frequencies. The coverage percentages, based on 1000 simulations, turned out to be close the desired values, namely $93.9 \%$, $93.5 \%, 94.8 \%$ and $94.8 \%$ for $n=512,1024,2048$ and 4096 respectively.

\subsection{El Niño and recruitment of new fish}

Figures $5 \mathrm{a}$ and $\mathrm{b}$ display the components of the bivariate time series consisting of the Southern Oscillation Index (SOI) and recruitment (amount) of new fish in the central Pacific Ocean (figures 5a and b), ranging from 1950 to 1987 over a period of $n=453$ months. The SOI relates changes in air pressure to the temperature of the ocean at the surface. The data set can be found in Shumway and Stoffer (2000). Both time series exhibit cyclic components. The dominating periodic component in the SOI has a period of 12 months. The second series oscillates with a lower frequency, but a 12-months cycle is visible as well, at least in parts of the series. This is most obvious when looking at the amplitude of the estimated regression cross spectrum in figure $5 \mathrm{c}$ which shows a dominating frequency at $j=38$ indicating a period of $453 / 38 \approx 12$ months. In addition, a certain number of moderate contributions are present at low frequencies. The slight influence of low frequency components is also visible directly in the SOI series, in that the mean and variability seem to be changing slowly. This feature is often 
refered to in the literature as the El Niño effect. We now proceed as in the simulated example. The estimated phase line in figure $5 \mathrm{f}$ is based on frequencies where the amplitude spectrum is large. The corresponding points are marked by black squares in figure 5e,f. Focussing on these points only, one can detect a linear structure. We may thus assume that there is a frequencyindependent shift $\Delta$. Linear regression yields a slope estimate of about 0.1 , and thus $\hat{\Delta}=0.1 /(2 \pi) \approx 0.016$ which corresponds to $453 \cdot 0.1 /(2 \pi) \approx 7.2$ months. This indicates that the SOI signal leads the recruitment of new fish by about seven to eight months.

However, in view of figure $5 \mathrm{c}$, better insight may be gained by separating high and low frequency components in the second series. We therefore carry out the analysis at separate levels of resolution. Figures $6 \mathrm{a}$ and $\mathrm{b}$ show trend estimates $\hat{f}_{1}$ (SOI) and $\hat{f}_{2}$ (fish recruitment) obtained by wavelet thresholding with s20-wavelets. The second trend function is decomposed further by separating the three coarsest resolution levels (figure 6c) from the fourth level (figure 6d). The fourth level represents the 12-month cycle, whereas the coarser parts (levels one to three, D4-S6) represent four to five year cycles that may be associated with corresponding cycles in the warming of the Pacific Ocean. The estimated amplitude cross spectrum with significant frequencies marked by black squares are displayed in figure 6e and the resulting phase line with corresponding confidence intervals is presented in figure $6 \mathrm{f}$. One notices again the distinct linear structure over this particular set of frequencies. The slope of this line is given by 0.17 indicating a lead of SOI of $453 \cdot 0.17 /(2 \pi) \approx 12$ months.

Because annual seasonality is the only common periodic oscillation between SOI and the component D3 (figure 6g), time delays between these two series are estimated in the time domain. The regression correlations between the SOI trend $f_{1}$ and the component D3 is displayed in figure $6 \mathrm{~h}$. The annual seasonality of the number of fish turns out to lag behind the SOI by about one month, indicating that an increased water temperature induces an increased number of fish about one month later. This effect interferes with the El Niño effect which has periods of abnormal warming of the sea every four to five years. These results confirm similar findings on the interplay between water temperature and fish recruitment by a number of authors such as Murawski (1993), Victor et al. (2001), Shumway and Stoffer (2000), Rosen and Stoffer (2007), among others. 


\section{Final remarks}

Analyzing multivariate dependence using the nonparametric regression spectrum is particularily useful when the observed series have strong deterministic components. In this article we defined a simple estimator of the regression spectrum based on the periodogram. In contrast to the method in Beran and Heiler (2007) no trend estimation is required. Also, in contrast to the stationary case, no smoothing of the periodogram is needed. In addition, lag estimation in the time domain was considered in order to be able to deal with cases where dependence between two series occurs for a small number of frequencies only. The regression spectrum approach can be particularily powerful when used in combination with multiresolution analysis. Often, the strength, type and interpretation of dependencies differ at different levels. The SOI/fish recruitment data is a typical example of multilevel dependence. In future research, more formal methods should be developed for combining regression spectrum estimation and wavelet decomposition.

\section{Acknowledgements}

This research was supported in part by a grant of the German Research Foundation (DFG).

\section{Appendix: Proofs}

Proof 1 (of theorem 1) We have $h_{r s}(j)=a_{r}(j) \overline{a_{s}(j)}$, and

$$
\frac{1}{n^{2}} A_{r}\left(\omega_{j}\right) \overline{A_{s}\left(\omega_{j}\right)}-h_{r s}(j)=O\left(n^{-1}\right)
$$

Then

$$
\begin{aligned}
& \frac{1}{n} I_{r s}\left(\omega_{j}\right)-h_{r s}(j) \\
& =\frac{1}{n^{2}}\left[B_{r}\left(\omega_{j}\right) \overline{A_{s}\left(\omega_{j}\right)}+A_{r}\left(\omega_{j}\right) \overline{B_{s}\left(\omega_{j}\right)}\right]+O_{p}\left(n^{-1}\right)+O\left(n^{-1}\right) \\
& =O_{p}\left(n^{-1 / 2}\right) .
\end{aligned}
$$


For the second part consider

$$
\begin{aligned}
E\left(n^{-1} I_{r s}\left(\omega_{j}\right)\right) & =\frac{1}{n^{2}}\left[A_{r}\left(\omega_{j}\right) \overline{A_{s}\left(\omega_{j}\right)}+E\left(B_{r}\left(\omega_{j}\right) \overline{B_{s}\left(\omega_{j}\right)}\right)\right], \\
& =\left(h_{r s}(j)+O\left(n^{-1}\right)\right)+n^{-1} E\left(I_{\epsilon ; r s}\left(\omega_{j}\right)\right) .
\end{aligned}
$$

Results from traditional spectral analysis show that $E\left(I_{\epsilon ; r s}\left(\omega_{j}\right)\right)$ converges to $2 \pi h_{\epsilon ; r s}\left(\omega_{j}\right)$ uniformly for all frequencies $\omega_{j}$ so that 2) follows.

Note furthermore that

$$
\frac{1}{\sqrt{n}}\left(a_{r}(j) \overline{B_{s}\left(\omega_{j}\right)}+B_{r}\left(\omega_{j}\right) \overline{a_{s}(j)}\right)=: \alpha(j)+i \beta(j),
$$

where

$$
\begin{aligned}
& \alpha(j)=\frac{1}{\sqrt{n}} \sum \cos \left(\omega_{j} t\right)\left[a_{r}(j) \epsilon_{s}(t)+\overline{a_{s}(j)} \epsilon_{r}(t)\right] \\
& \beta(j)=\frac{1}{\sqrt{n}} \sum \sin \left(\omega_{j} t\right)\left[a_{r}(j) \epsilon_{s}(t)-\overline{a_{s}(j)} \epsilon_{r}(t)\right] .
\end{aligned}
$$

Consider now the variance of the adjusted periodogram (the result for covariances follows similarly):

$$
\begin{aligned}
\operatorname{var}\left(n^{-1} I_{r s}\left(\omega_{j}\right)\right) & =n^{-1} E\left[|\alpha(j)+i \beta(j)|^{2}\right] \\
& +\operatorname{cov}\left(n^{-1} I_{\epsilon ; r s}\left(\omega_{j}\right), \alpha(j)+i \beta(j)\right) \\
& +\operatorname{cov}\left(\alpha(j)+i \beta(j), n^{-1} I_{\epsilon ; r s}\left(\omega_{j}\right)\right) \\
& +\operatorname{var}\left(n^{-1} I_{\epsilon ; r s}\left(\omega_{j}\right)\right) .
\end{aligned}
$$

Using standard results from Brockwell and Davis (p.429) yields

$\operatorname{cov}\left(I_{\epsilon ; r s}\left(\omega_{j}\right), I_{\epsilon ; r s}\left(\omega_{j^{\prime}}\right)\right)=\left\{\begin{array}{lr}\Sigma_{r r} \Sigma_{s s}+O\left(n^{-1}\right) ; & 0<\omega_{j}=\omega_{j^{\prime}}<\pi, \\ \Sigma_{r r} \Sigma_{s s}+\Sigma_{r s} \Sigma_{s r}+O\left(n^{-1}\right) ; & \omega_{j}=\omega_{j^{\prime}} \in\{0, \pi\}, \\ O\left(n^{-1}\right) ; & \omega_{j} \neq \omega_{j^{\prime}},\end{array}\right.$

where the remainders contain the fourth order cumulants between $\epsilon_{r}(i)$ and $\epsilon_{s}(i)$. The covariances in (21) and (22) consist of terms of the form

$$
\begin{aligned}
\operatorname{cov}\left(I_{\epsilon ; r s}\left(\omega_{j}\right), B_{r}\left(\omega_{j}\right)\right) & =\frac{1}{n} \sum_{t, u, v} \exp \left(-i \omega_{j}(t-u+v)\right) E\left(\epsilon_{r}(t) \epsilon_{s}(u) \epsilon_{r}(v)\right) \\
& =\frac{E\left(\epsilon_{r}(1)^{2} \epsilon_{s}(1)\right)}{n} \sum_{t=1}^{n} \exp \left(i \omega_{j} t\right)=0,
\end{aligned}
$$


where $E\left(\epsilon_{r}(t)^{2} \epsilon_{s}(t)\right)$ is independent of $t$. Simple considerations show that the $2 p$-dimensional real valued random vector

$$
\mathbf{U}_{\mathbf{n}}\left(\omega_{j}\right)=n^{-1 / 2}\left(\begin{array}{c}
\sum \boldsymbol{\epsilon}(t) \cos \left(\omega_{j} t\right) \\
\sum \boldsymbol{\epsilon}(t) \sin \left(\omega_{j} t\right)
\end{array}\right)
$$

is asymptotically normal with mean $\mathbf{0}$ and covariance matrix

$$
E\left(\mathbf{U}_{\mathbf{n}}\left(\omega_{j}\right) \mathbf{U}_{\mathbf{n}}\left(\omega_{j}\right)^{T}\right)=\frac{1}{2}\left(\begin{array}{cc}
\boldsymbol{\Sigma} & 0 \\
0 & \boldsymbol{\Sigma}
\end{array}\right) .
$$

Furthermore, for all Fourier frequencies $\omega_{j} \neq \omega_{j^{\prime}}$,

$$
E\left(\mathbf{U}_{\mathbf{n}}\left(\omega_{j}\right) \mathbf{U}_{\mathbf{n}}\left(\omega_{j^{\prime}}\right)^{T}\right)=0 .
$$

Therefore,

$$
\begin{aligned}
& n^{-1} E\left(B_{r}\left(\omega_{j}\right) \overline{B_{s}\left(\omega_{j}\right)}\right) \\
& =n^{-1} \operatorname{cov}\left(\sum \epsilon_{r}(t)\left(\cos \left(\omega_{j} t\right)-i \sin \left(\omega_{j} t\right)\right), \sum \epsilon_{s}(t)\left(\cos \left(\omega_{j} t\right)-i \sin \left(\omega_{j} t\right)\right)\right) \\
& =\frac{1}{2}\left(\Sigma_{r s}+\Sigma_{s r}\right)=\Sigma_{r s} .
\end{aligned}
$$

Similarly, for all pairs $(r, s), 1 \leq r \neq s \leq p$,

$$
\begin{aligned}
\operatorname{var}\left(n^{-1 / 2} B_{r}\left(\omega_{j}\right)\right) & =\Sigma_{r r} \quad \text { and } \\
\operatorname{cov}\left(B_{r}\left(\omega_{j}\right), \overline{B_{s}\left(\omega_{j}\right)}\right) & =\operatorname{cov}\left(\overline{B_{s}\left(\omega_{j}\right)}, B_{r}\left(\omega_{j}\right)\right)=0 .
\end{aligned}
$$

Noting that

$$
\sum_{t=1}^{n} \cos ^{2}\left(\omega_{j} t\right)=\frac{n}{2},
$$

the variance in (20) follows from

$$
\begin{aligned}
\tau_{\alpha}^{2}(j):= & \operatorname{var}(\alpha(j)) \\
= & \frac{1}{n} \sum_{t=1}^{n} \cos ^{2}\left(\omega_{j} t\right)\left[\Sigma_{r r}\left|a_{s}(j)\right|^{2}+\Sigma_{s s}\left|a_{r}(j)\right|^{2}\right. \\
& \left.\quad+\overline{a_{s}(j) a_{r}(j)} \operatorname{cov}\left(\epsilon_{r}(t), \epsilon_{s}(t)\right)+a_{r}(j) a_{s}(j) \operatorname{cov}\left(\epsilon_{s}(t), \epsilon_{r}(t)\right)\right] \\
= & \frac{1}{2}\left(\Sigma_{r r}\left|a_{s}(j)\right|^{2}+\Sigma_{s s}\left|a_{r}(j)\right|^{2}\right)+\Sigma_{r s} \mathfrak{R e}\left\{a_{s}(j) a_{r}(j)\right\}
\end{aligned}
$$


Similarily,

$$
\tau_{\beta}^{2}(j):=\operatorname{var}(\beta(j))=\frac{1}{2}\left(\Sigma_{r r}\left|a_{s}(j)\right|^{2}+\Sigma_{s s}\left|a_{r}(j)\right|^{2}\right)-\Sigma_{r s} \mathfrak{R e}\left\{a_{s}(j) a_{r}(j)\right\}
$$

and

$$
\begin{aligned}
& \tau_{\alpha, \beta}(j):=\operatorname{cov}(\alpha(j), \beta(j)) \\
= & \frac{1}{n} \sum_{t=1}^{n} \cos \left(\omega_{j} t\right) \sin \left(\omega_{j} t\right) \operatorname{cov}\left(\overline{a_{s}(j)} \epsilon_{r}(t)+a_{r}(j) \epsilon_{s}(t), a_{r}(j) \epsilon_{s}(t)-\overline{a_{s}(j)} \epsilon_{r}(t)\right),
\end{aligned}
$$

where the covariance is independent of $t$. Using the orthogonality relations for trigonometric functions we get that $\tau_{\alpha, \beta}(j)=0$.

For the asymptotic distribution, write

$$
\sqrt{n}\left(\frac{1}{n} I\left(\omega_{j}\right)-h_{r s}(j)\right)=\alpha(j)+i \beta(j)+O_{p}\left(n^{-1 / 2}\right) .
$$

According to (24) the asymptotic distribution of the real and the imaginary part are both univariate normal. Moreover, $\alpha(j)$ and $\beta(j)$ are uncorrelated and hence asymptotically independent. Hence,

$$
\left(\begin{array}{c}
\alpha(j) \\
\beta(j)
\end{array}\right) \stackrel{d}{\rightarrow} \mathcal{N}\left(\mathbf{0},\left[\begin{array}{cc}
\tau_{\alpha}^{2}(j) & 0 \\
0 & \tau_{\beta}^{2}(j)
\end{array}\right]\right),
$$

and $\alpha(j)+i \beta(j)$ converges in distribution to a complex valued normal random variable with mean 0 and variance

$$
\tau_{\alpha+i \beta}^{2}(j)=\tau_{\alpha}^{2}(j)+\tau_{\beta}^{2}(j)=\Sigma_{r r}\left|a_{s}(j)\right|^{2}+\Sigma_{s s}\left|a_{r}(j)\right|^{2} .
$$

This implies

$$
\sqrt{n}\left(\frac{1}{n} I_{r s}\left(\omega_{j}\right)-h_{r s}(j)\right) \stackrel{d}{\rightarrow} \mathcal{N}_{c}\left(0, \Sigma_{r r}\left|a_{s}(j)\right|^{2}+\Sigma_{s s}\left|a_{r}(j)\right|^{2}\right) .
$$

The result for finite samples $\left(\omega_{j_{1}}, \ldots, \omega_{j_{k}}\right)$ follows as usually by applying the Cramer-Wold device.

Proof 2 (of theorem 2)

The following lemma summarizes asymptotic results from Hannan (1970). 
Lemma 2 Assume that the sequence $\{\boldsymbol{\epsilon}(i)\}, i=1, \ldots, n$, is of the form

$$
\boldsymbol{\epsilon}(i)=\sum_{j=-\infty}^{\infty} \mathbf{A}(j) \mathbf{Z}(i-j)
$$

where $\mathbf{A}(j)=\left(A_{l k}(j)\right)_{1 \leq l, k \leq p}$ are $p \times p$-matrices such that for all pairs $(l, k)$,

$$
\sum_{j \in \mathbb{Z}}\left|A_{l k}(j)\right||j|^{\frac{1}{2}}<\infty
$$

and the sequence $\mathbf{Z}(i)$ is independent and identically distributed with mean 0 and non-singular covariance matrix $\mathbf{\Sigma}$.

Then,

$$
\mathbf{g}\left(\omega_{j}\right)=n^{-1 / 2}\left(\begin{array}{c}
\sum \boldsymbol{\epsilon}(t) \cos \left(\omega_{j} t\right) \\
\sum \boldsymbol{\epsilon}(t) \sin \left(\omega_{j} t\right)
\end{array}\right)=\left(\begin{array}{l}
\mathbf{g}_{1}\left(\omega_{j}\right) \\
\mathbf{g}_{2}\left(\omega_{j}\right)
\end{array}\right)
$$

with $\mathbf{g}_{l}\left(\omega_{j}\right)=\left(g_{l 1}\left(\omega_{j}\right), \ldots, g_{l p}\left(\omega_{j}\right)\right)^{T}, l \in\{1,2\}$, converges in distribution to a $2 p$-dimensional random variable with mean $\mathbf{0}$ and asymptotic covariance matrix

$$
\pi\left(\begin{array}{cc}
\mathbf{C}\left(\omega_{j}\right) & \mathbf{Q}\left(\omega_{j}\right) \\
-\mathbf{Q}\left(\omega_{j}\right) & \mathbf{C}\left(\omega_{j}\right)
\end{array}\right)
$$

where $\mathbf{h}_{\epsilon}\left(\omega_{j}\right)=\frac{1}{2}\left(\mathbf{C}\left(\omega_{j}\right)-i \mathbf{Q}\left(\omega_{j}\right)\right)$ with $\mathbf{C}\left(\omega_{j}\right)=\left[c_{\epsilon ; r s}\left(\omega_{j}\right)\right]_{1 \leq r, s \leq p}$ and $\mathbf{Q}\left(\omega_{j}\right)=$ $\left[q_{\epsilon ; r s}\left(\omega_{j}\right)\right]_{1<r, s \leq p}$ is the spectral density matrix of $\boldsymbol{\epsilon}(i)$ at Fourier frequency $\omega_{j}=2 \pi j / n$. Furthermore $g\left(\omega_{j}\right)$ and $g\left(\omega_{j^{\prime}}\right)$ are asymptotically independent for $\omega_{j} \neq \omega_{j^{\prime}}$.

We now turn to the proof of theorem 2. The first two parts of the proof are similar to those of theorem 1. For the asymptotic distribution and variance consider equations (20)-(23). Refer again to Brockwell and Davis (p. 431) to get

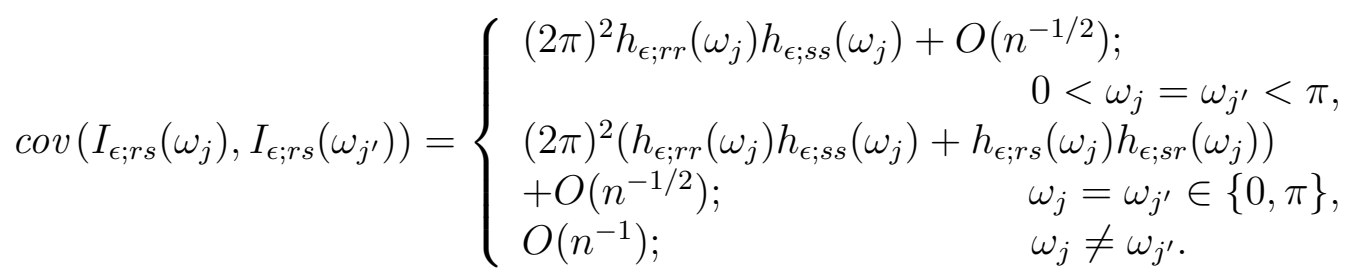


Using the results of lemma 2 ,

$$
\operatorname{var}\left(\frac{1}{\sqrt{n}} B_{r}\left(\omega_{j}\right)\right)=2 \pi h_{\epsilon ; r r}\left(\omega_{j}\right) \quad(1 \leq r \leq p)
$$

and use the notation

$$
h_{\epsilon ; r r}\left(\omega_{j}\right)=c_{\epsilon ; r s}\left(\omega_{j}\right)-i q_{\epsilon ; r s}\left(\omega_{j}\right) .
$$

Then,

$$
\begin{aligned}
& \operatorname{cov}\left(\frac{1}{\sqrt{n}} B_{r}\left(\omega_{j}\right), \frac{1}{\sqrt{n}} \overline{B_{s}\left(\omega_{j}\right)}\right) \\
& =\operatorname{cov}\left(g_{1 r}\left(\omega_{j}\right)-i g_{2 r}\left(\omega_{j}\right), g_{1 s}\left(\omega_{j}\right)+i g_{2 s}\left(\omega_{j}\right)\right) \\
& =c_{\epsilon ; r s}\left(\omega_{j}\right)+i q_{\epsilon ; r s}\left(\omega_{j}\right)-i q_{\epsilon ; r s}\left(\omega_{j}\right)-c_{\epsilon ; r s}\left(\omega_{j}\right)=0 .
\end{aligned}
$$

Due to lemma 2 and equations (29) and (30),

$$
\alpha(j)+i \beta(j) \stackrel{d}{\rightarrow} \mathcal{N}_{c}\left(0,\left(2 \pi\left|a_{s}(j)\right|^{2} h_{\epsilon ; r r}\left(\omega_{j}\right)+2 \pi\left|a_{r}(j)\right|^{2} h_{\epsilon ; s s}\left(\omega_{j}\right)\right)\right),
$$

where $\alpha(j)+i \beta(j)$ is defined in (17). The remaining parts (21) and (22) consist of terms of the form

$$
\operatorname{cov}\left(I_{\epsilon ; r s}\left(\omega_{j}\right), B_{r}\left(\omega_{j}\right)\right)=\sum_{t, u, v} \exp \left(-i \omega_{j}(t-u+v)\right) E\left(\epsilon_{r}(t) \epsilon_{s}(u) \epsilon_{r}(v)\right)
$$

with

$$
\begin{aligned}
& E\left(\epsilon_{r}(t) \epsilon_{s}(u) \epsilon_{r}(v)\right) \\
& =\sum_{r_{1}, r_{2}, r_{3}=1}^{p} E\left(Z_{r_{1}}(1) Z_{r_{2}}(1) Z_{r_{3}}(1)\right) \sum_{j} A_{r, r_{1}}(j) A_{s, r_{2}}(j+u-t) A_{r, r_{3}}(j+v-t),
\end{aligned}
$$

where $A_{r r_{1}}(j)$ is the $\left(r, r_{1}\right)$ th entry of the matrix $\mathbf{A}(j)$ and $E\left(Z_{r_{1}}(t) Z_{r_{2}}(t) Z_{r_{3}}(t)\right)$ is the third order cumulant between the components $Z_{r_{1}}(t), Z_{r_{2}}(t)$ and $Z_{r_{3}}(t)$ of $\mathbf{Z}(t)$. Because $\boldsymbol{\epsilon}(i)$ is of the form (28),

$$
\left|\operatorname{cov}\left(I_{\epsilon ; r s}\left(\omega_{j}\right), B_{r}\left(\omega_{j}\right)\right)\right| \leq \sum_{t, u, v}\left|E\left(\epsilon_{r}(t) \epsilon_{s}(u) \epsilon_{r}(v)\right)\right|<\infty
$$

so that $\tau_{n}\left(\omega_{j}\right)=O\left(n^{-1}\right)$. 


\section{Proof 3 (of Theorem 3)}

1. Results of Brillinger (1996) prove wavelet thresholding estimators to be consistent of order $O_{p}\left(n^{-1 / 2}\right)$. The regression cross covariance is a continuous function of $\theta$, the vector of wavelet coefficient of $f_{r}$ and $f_{s}$, so that the estimator $\hat{u}_{r s}^{\text {max }}$ is a continuous function of $\hat{\theta}$. Consistency of $\hat{\theta}$ then implies consistency of $u_{r s}^{\max }$.

2. Due to (14) and (15),

$$
\gamma_{r s}^{\prime}\left(\hat{u}_{r s}^{\max }, \hat{\theta}\right)=0 \text { and } \gamma_{r s}^{\prime}\left(u_{r s}^{\max }, \theta_{0}\right)=0 .
$$

Applying the mean value theorem gives

$$
\gamma_{r s}^{\prime}\left(\hat{u}_{r s}^{\max }, \hat{\theta}\right)-\gamma_{r s}^{\prime}\left(u_{r s}^{\max }, \hat{\theta}\right)=\gamma_{r s}^{\prime \prime}\left(\tilde{u}_{r s}, \hat{\theta}\right)\left(\hat{u}_{r s}^{\max }-u_{r s}^{\max }\right),
$$

where $\tilde{u}$ lies on a line connecting $\hat{u}_{r s}^{\max }$ and $u_{r s}^{\max }$. Therefore,

$$
\hat{u}_{r s}^{\max }-u_{r s}^{\max }=-\frac{\gamma_{r s}^{\prime}\left(u_{r s}^{\max }, \hat{\theta}\right)}{\gamma_{r s}^{\prime \prime}\left(\tilde{u}_{r s}, \hat{\theta}\right)} .
$$

Furthermore,

$$
\gamma_{r s}^{\prime}\left(u_{r s}^{\max }, \hat{\theta}\right)=\underbrace{\gamma_{r s}^{\prime}\left(u_{r s}^{\max }, \theta_{0}\right)}_{=0}+\left(\left.\frac{\partial}{\partial \theta} \gamma_{r s}^{\prime}\left(u_{r s}^{\max }, \theta\right)\right|_{\theta=\tilde{\theta}}\right)^{T} \cdot\left(\hat{\theta}-\theta_{0}\right)
$$

for suitably chosen $\tilde{\theta}$, and

$$
\hat{u}_{r s}^{\max }-u_{r s}^{\max }=-\frac{1}{\gamma_{r s}^{\prime \prime}\left(\tilde{u}_{r s}, \hat{\theta}\right)}\left(\left.\frac{\partial}{\partial \theta} \gamma_{r s}^{\prime}\left(u_{r s}^{\max }, \theta\right)\right|_{\theta=\tilde{\theta}}\right)^{T}\left(\hat{\theta}-\theta_{0}\right) .
$$

Due to consistency of $\hat{\theta}$ and $\hat{u}_{r s}^{\max }$,

$$
\begin{aligned}
\gamma_{r s}^{\prime \prime}\left(\tilde{u}_{r s}, \hat{\theta}\right) & =\gamma_{r s}^{\prime \prime}\left(u_{r s}^{\max }, \theta_{0}\right)+\left(\gamma_{r s}^{\prime \prime}\left(\tilde{u}_{r s}, \hat{\theta}\right)-\gamma_{r s}^{\prime \prime}\left(u_{r s}^{\max }, \hat{\theta}\right)\right) \\
& +\left(\gamma_{r s}^{\prime \prime}\left(u_{r s}^{\max }, \hat{\theta}\right)-\gamma_{r s}^{\prime \prime}\left(u_{r s}^{\max }, \theta_{0}\right)\right) \\
& =\gamma_{r s}^{\prime \prime}\left(u_{r s}^{\max }, \theta_{0}\right)+O_{p}\left(n^{-1 / 2}\right),
\end{aligned}
$$

and

$$
\left.\frac{\partial}{\partial \theta} \gamma_{r s}^{\prime}\left(u_{r s}^{\max }, \theta\right)\right|_{\theta=\tilde{\theta}}=\left.\frac{\partial}{\partial \theta} \gamma_{r s}^{\prime}\left(u_{r s}^{\max }, \theta\right)\right|_{\theta=\theta_{0}}+O_{p}\left(n^{-1 / 2}\right)
$$


Then,

$\hat{u}_{r s}^{\max }-u_{r s}^{\max }=-\frac{1}{\gamma_{r s}^{\prime \prime}\left(u_{r s}^{\max }, \theta_{0}\right)}\left(\frac{\partial}{\partial \theta} \gamma_{r s}^{\prime}\left(u_{r s}^{\max }, \theta_{0}\right)\right)^{T}\left(\hat{\theta}-\theta_{0}\right)\left(1+O_{p}\left(n^{-1 / 2}\right)\right)$.

The central limit theorem for $\sqrt{n}\left(\hat{\theta}-\theta_{0}\right)$ implies that $\sqrt{n}\left(\hat{u}_{r s}^{\max }-u_{r s}^{\max }\right)$ is asymptotically normal with mean 0 and covariance

$$
\operatorname{var}\left(\hat{u}_{r s}^{\max }\right)=\frac{\left(\left.\frac{\partial}{\partial \theta} \gamma_{r s}^{\prime}\left(u_{r s}^{\max }, \theta\right)\right|_{\theta=\theta_{0}}\right)^{T} \operatorname{var}(\hat{\theta})\left(\left.\frac{\partial}{\partial \theta} \gamma_{r s}^{\prime}\left(u_{r s}^{\max }, \theta\right)\right|_{\theta=\theta_{0}}\right)}{\gamma_{r s}^{\prime \prime}\left(u_{r s}^{\max }, \theta_{0}\right)^{2}} .
$$

\section{References}

[1] Beran, J. and Ghosh, S. (2000). Estimation of the dominating frequency for stationary and nonstationary fractional autoregressive models. Journal of Time Series Analysis, Vol. 21, No. 5, 517-533.

[2] Beran, J. and Heiler, M. (2007). A Nonparametric Regression Spectrum for Analyzing Multivariaten Time Series. Journal of Multivariate Analysis, in press.

[3] Brillinger, D. (1994). Uses of Cumulants in Wavelet Analysis. Proc. SPIE, Advanced Signal Processing, Vol. 2296, 2-18.

[4] Brillinger, D.R. (1996). Some Uses of Cumulants in Wavelet Analysis. Nonparametric Statistics, Vol. 6, 93-114.

[5] Brockwell, P.J, and Davis, R.A. (1987). Time Series: Theory and Methods. Springer, New York.

[6] Daubechies, I. (1999). Ten Lectures on Wavelets. Society for Industrial and Applied Mathematics, Philadelphia.

[7] Donoho, D.L. and Johnstone, I.M. (1994). Ideal Spatial Adaption by Wavelet Shrinkage. Biometrika, Vol. 81, No.3.

[8] Donoho, D.L. and Johnstone, I.M. (1995). Adapting to Unknown Smoothness Via Wavelet Shrinkage. Journal of the American Statistical Association, Vol. 90. 
[9] Grenander, U. and Rosenblatt, M. (1957). Statistical analysis of stationary time series, Wiley, New York.

[10] Hannan, E.J. (1970). Multiple time series. Wiley, New York.

[11] Heiler, M. (2007). A Nonparametric Regression Spectrum: Estimation, Asymptotic Properties and Data Analysis. Dissertation, University of Konstanz, Konstanz, 2007.

[12] Johnstone, I.M. and Silverman, B.W. (1997). Wavelet Threshold Estimators for Data with Correlated Noise. Journal of the Royal Statistical Society, Series B, Vol. 59, No. 2, 319-351.

[13] Murawski, S.A. (1993). Climate change and marine fish distributions: forecasting from analogy. Transactions of the American Fisheries Society, Vol. 122, 647-658.

[14] Percival, D.P. and Walden, A.T. (2000). Wavelet methods for time series analysis. Cambridge University Press, Cambridge.

[15] Pollard, D. (1984). Convergence of Stochastic Processes. $2^{\text {nd }}$ Edition, Springer, New York.

[16] Polya G. and Szegö G. (1964). Aufgaben und Lehrsätze aus der Analysis, Erster Band, 3. Auflage. Springer, Berlin.

[17] Priestley, M.B. (1989). Spectral Analysis and Time Series, $6^{\text {th }}$ Edition, Academic Press, London.

[18] Rosen, O. and Stoffer, D.S. (2007). Automatic estimation of multivariate spectra via smoothing splines. Biometrika, 94, No. 2, 335-345.

[19] Shumway, R.H. and Stoffer, D.S. (2000). Time series analysis and its applications, $2^{\text {nd }}$ Edition. Springer, New York.

[20] Victor, B.C., Wellington, G.M., Robertson, D.R. and Ruttenberg, B.I. (2001). The effect of the EL Niño-Southern Oscillation event on the distribution of reef-associated labrid fishes in the eastern Pacific Ocean. Bulletin of Marine Science, Vol. 69, No. 1, 280-288. 

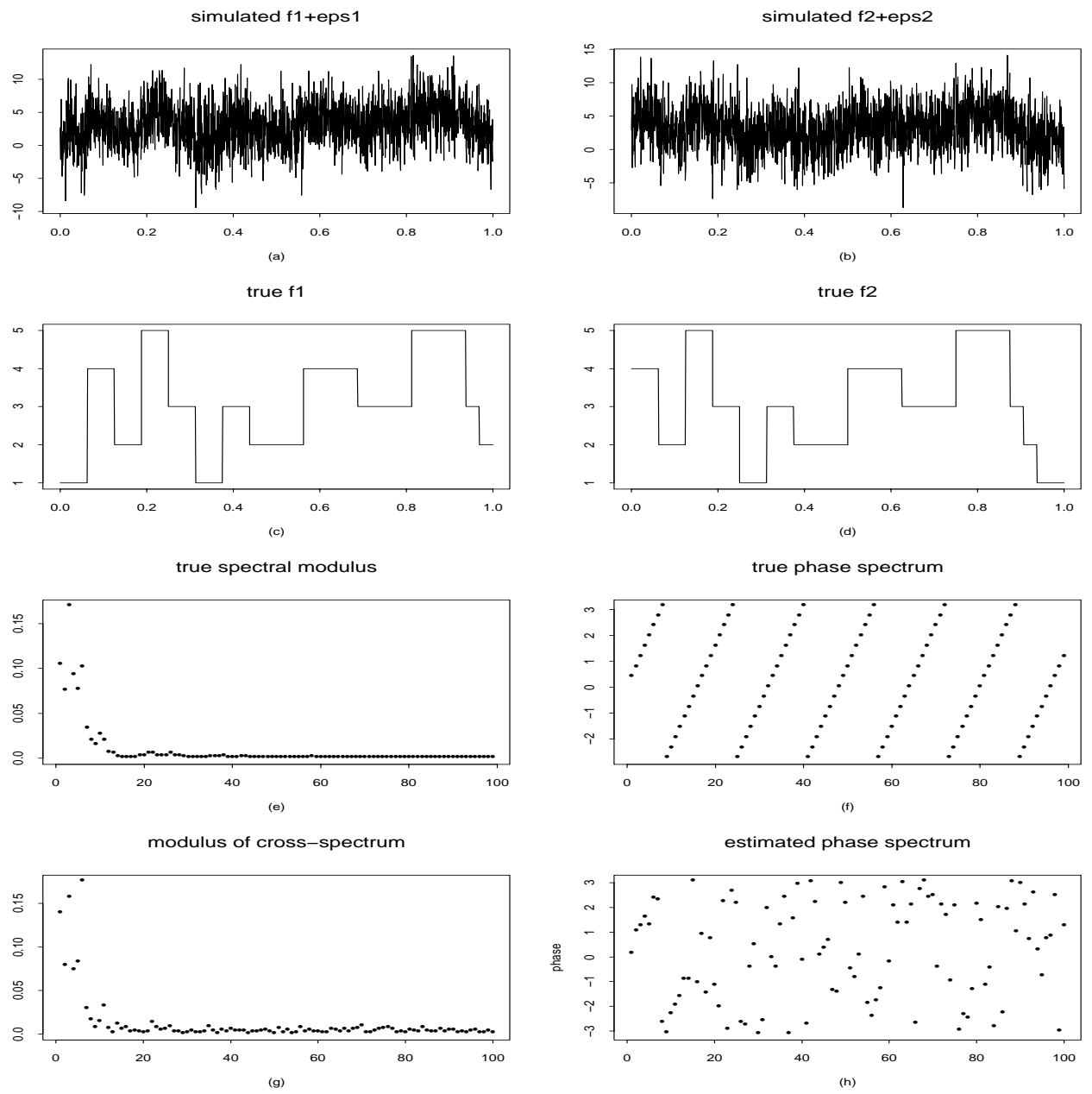

Figure 1: Simulated series of length $n=2048$ (figures 1a,b), as defined in section 5.1, trend components (figures 1c,d), and amplitude and phase of the true regression spectrum (figures 1e,f). Estimates of the amplitude and phase spectrum based on the periodogram are given figures $1 \mathrm{~g}$ and $\mathrm{h}$. 

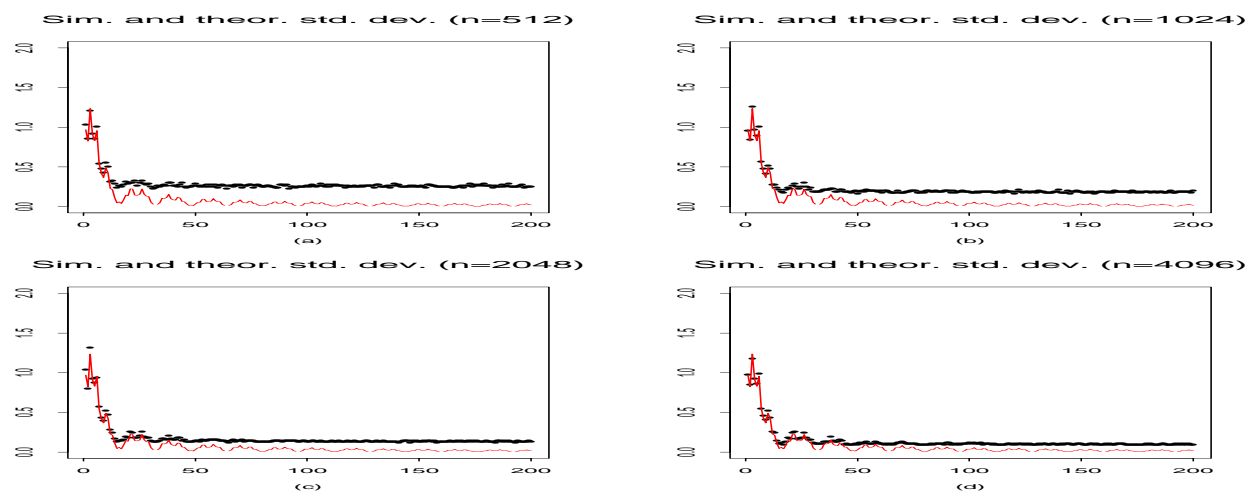

Figure 2: Simulated and theoretical (red line) standard deviations for the amplitude spectrum for various sample sizes $n=512,1024,2048$ and $n=4096$. For each sample size, 200 simulations were carried out at each individual frequency.
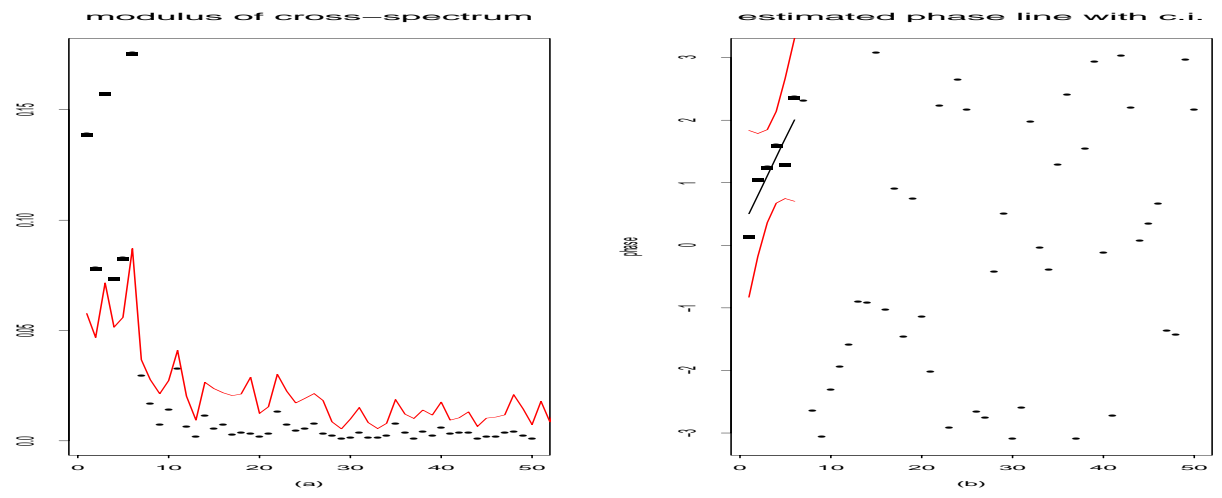

Figure 3: Estimates of the amplitude and phase spectrum based on the periodogram for frequencies $j=1, \ldots, 50$. Important common frequencies are marked by black squares. The estimated phase line (solid line) is based on these frequencies. The red lines represent $99 \%$ confidence intervals. 


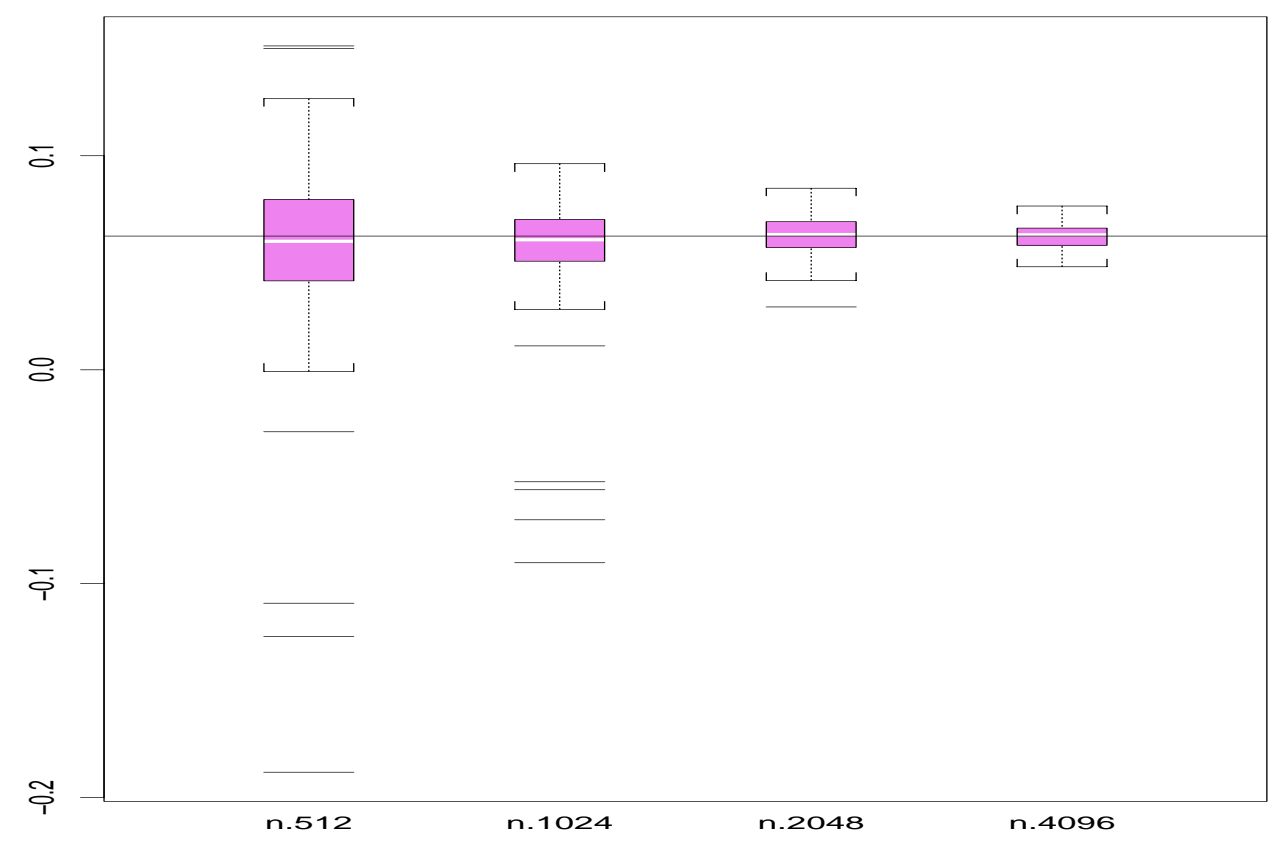

Figure 4: Boxplots of simulated lag estimates for $n=512,1024,2048$ and $n=4096$. For each sample size, 200 simulations were carried out. The true value 0.0625 is marked by a horizontal line. 

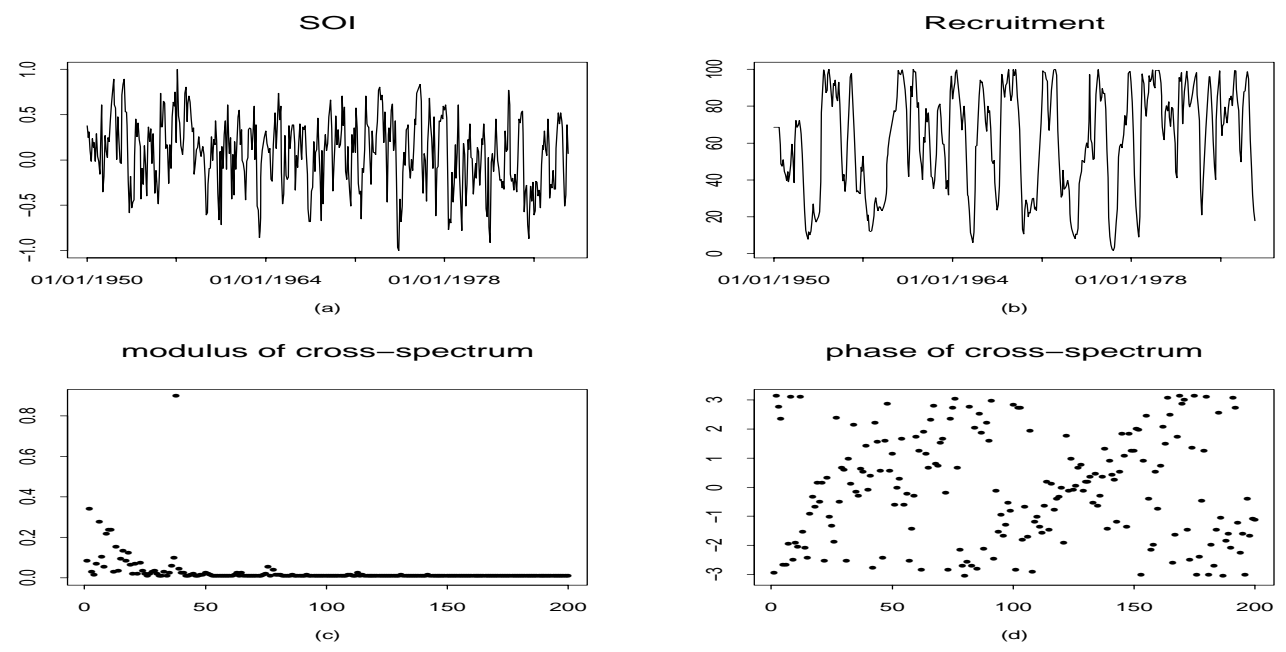

modulus of cross-spectrum
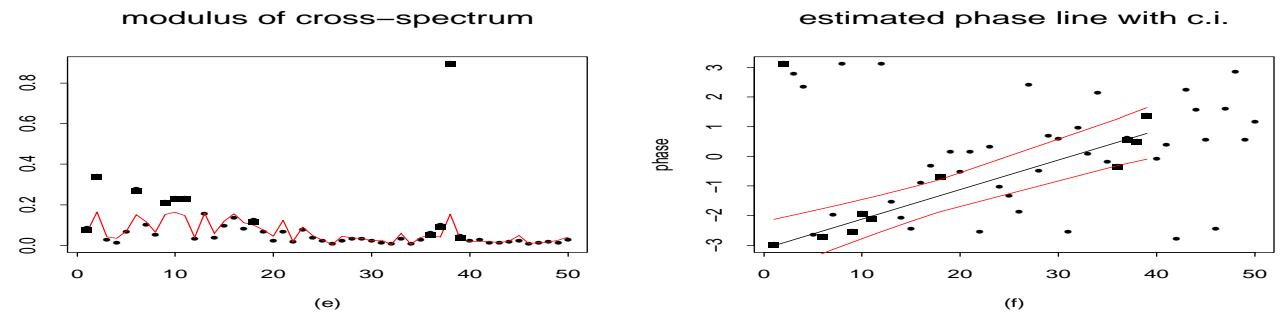

Figure 5: Monthly SOI and numbers of new fish (figures 5a,b) for the period 1950-1987, estimated amplitude of cross-spectrum (figures 5c,d), cross amplitude spectrum (figure 5e) with line representing three times the standard deviation and phase spectrum (figure 5f). Important frequencies are marked in both plots by black squares. Estimation of the phase line is based on these frequencies. The red lines in figure 5 f represent $99 \%$ confidence intervals for the regression line. 

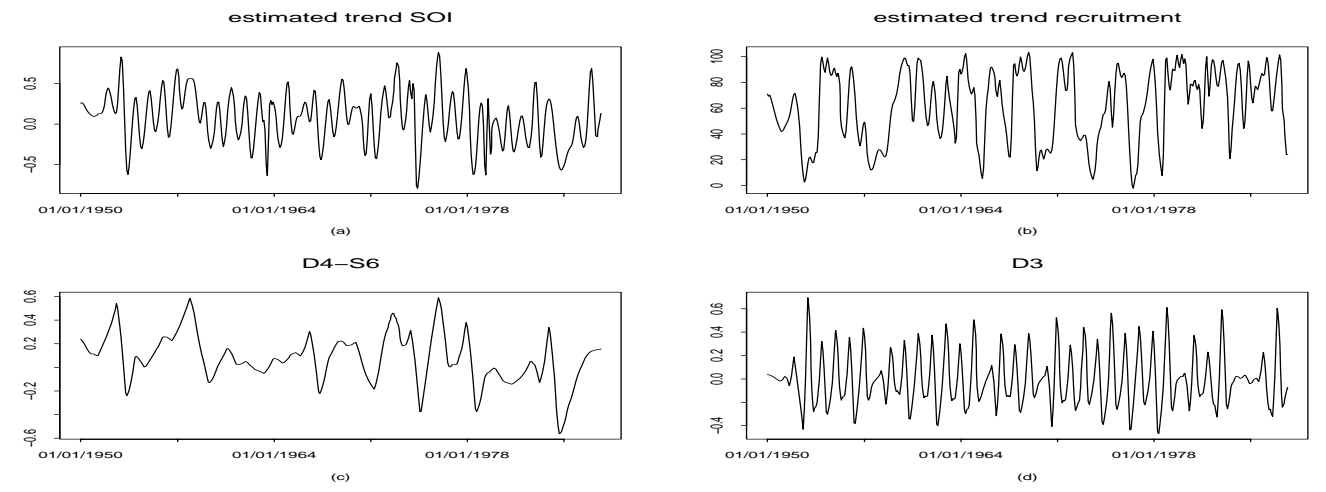

modulus (D4-S6)
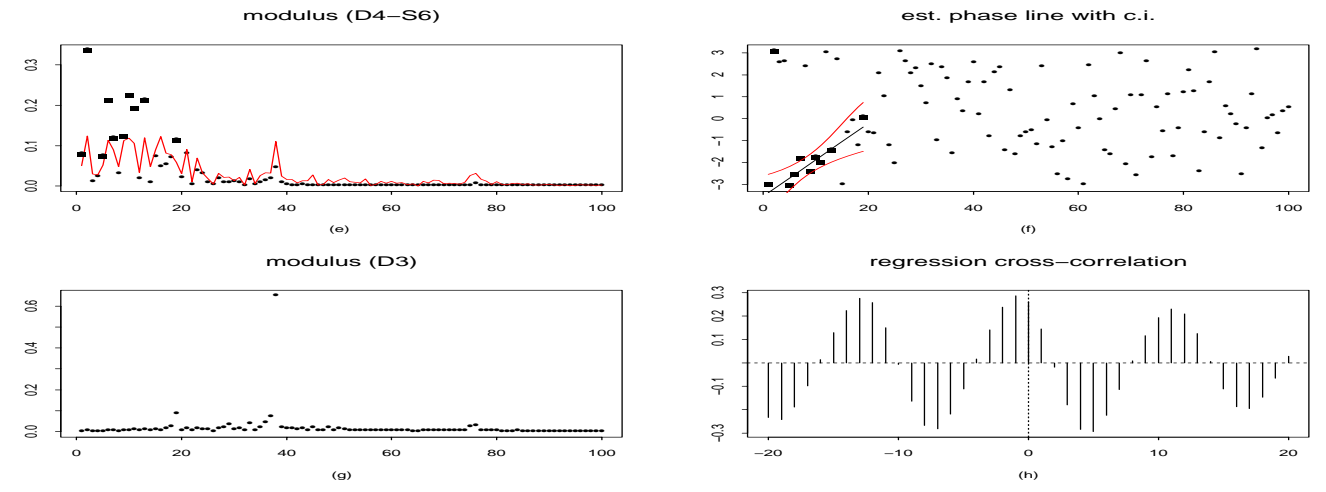

Figure 6: Wavelet estimates of trend components of SOI and the recruitment of new fish are displayed in figure $6 \mathrm{a}$ and figure $6 \mathrm{~b}$. The series in $6 \mathrm{~b}$ is then decomposed into a long-term component (figure 6c) given by the three coarsest levels of wavelet decomposition and a 12-months cycle corresponding to the fourth resolution level (figure 6d). The amplitude of cross-spectrum of D4-S6 with SOI is represented in figure 6e with the solid line given by three times the standard deviation. Important frequencies are again marked by black squares. The estimated phase line in figure $6 \mathrm{f}$ is based on these frequencies. The level D3 essentially corresponds to a 12-month cycle which is represented by a dominating frequency at $j=38$ (figure 6e). Estimation of the lag by periodogram analysis is inappropriate based on a single frequency. Therefore, the cross-correlations of the D3-component is displayed in figure $6 f$. 